\title{
Stable long term avoidance responding and fixed ratio avoidance training
}

\author{
Pietro Badia, BOWLING GREEN STATE UNIVERSITY \\ Sheldon Levine, OHIO STATE UNIVERSITY
}

\begin{abstract}
Two studies are reported dealing with avoidance conditioning in rats. In the first study the stability of the shuttle response was investigated in 4 Ss running $2 \mathrm{hrs}$. a day over an 18 day period. Stable long term avoidance responding was obtained. Except for extinction, no decrement in avoidance responding developed at anytime. In the second study, fixed ratio reinforcement of the avoidance response was attempted in $10 \mathrm{Ss}$. Eight Ss acquired the continuously reinforced avoidance response; 2 Ss reached a 2:1 ratio criterion; 4 Ss acquired a 4:1 ratio, and 2 Ss went through a $7: 1$ and 10:1 ratio. One of the latter Ss attained a fixed ratio of $21: 1$.

\section{Introduetion}

One of the main interests of this research is determining the feasibility of intermittently reinforcing an avoidance response. However, since the study of intermittent reinforcement typically requires a long training period, consideration must first be given to the difficulties involved in giving extended avoidance conditioning. For example, Coons et al., (1960) report the disappearance of a wheel turning avoidance response with continued training. Employing a different class of avoidance responses could possibly avoid this problem. But, as reported by Meyer et al., (1960) some responses, such as a bar pressing response, may create equally distressing problems during acquisition.
\end{abstract}

\section{Study 1}

A commonly used easily acquired avoidance response is the shuttle response. There is little information available, however, dealing with the limitations and stability of this response over an extended training period. The purpose of Study 1 is to provide this information. Method

Four female hooded rats between the ages of 50 and 90 days served as Ss in a shuttle box ( $24 \times 10 \times 8$ in) wired for scrambled shock. A 3 in barrier with an independently controlled shock source divided the box into halves. Two light sources with infrared filters 2 in to the sides of the barrier in combination with photocells and relays controlled the circuitry.

Shock avoidance training was given using a delayed conditioning procedure with a 10 sec. CS-US interval and a one min. intertrial interval. A hurdle jumpduring the $10 \mathrm{sec}$. interval resulted in termination of the CS and prevention of the US. Failure to respond during the 10 sec. interval resulted in the US being presented; a hurdle jump during this period terminated both CS and US. If an escape or an avoidance response did not occur shock was terminated at the end of a $60 \mathrm{sec}$. period. A compound CS consisted of a 24 volt buzzer ( $65 \mathrm{db})$ and two 110 volt $11 / 2$ watt lights mounted on the ends of the box. The US was a 40 volt AC shock passed through 100,000 ohms resistance. Two hours training was given each $\mathrm{S}$ for 18 days. At the end of this period 2 Ss were given extinction training.

\section{Results}

All Ss were successfully avoiding by the second hour of the first day's session and continued avoiding for the duration of the experiment. It is quite clear that the shuttle response can be maintained over long periods since no marked response loss or decremental avoidance tendency appeared with continued training. With the exception of extinction trials, no Ss dropped below $80 \%$ avoidance. These extinction data rule out the possibility of pseudoconditioning or CS aversiveness.

\section{Study 2}

In contrast to the findings of Meyer et al. (1960), the previous study showed that stable long term avoidance responding can be maintained, at least with a shuttle response. It therefore appears feasible to investigate variables that typically require extended training periods. Fixed ratio reinforcement of the avoidance response was therefore attempted in Study 2 .

The training period for learning of the avoidance response in Study 1 was such that the termination of the CS and the withholding of the aversive US was dependent upon the occurrence of a single response. For

$$
\text { Table I }
$$

Mean Latency and Mean Time per Shuttle

$$
\text { for } 2 \text { Ss Attaining a 10:1 }
$$

\begin{tabular}{|c|c|c|c|}
\hline & \multicolumn{2}{|c|}{ Ratios } & \\
\hline & $4: 1$ & $7: 1$ & $10: 1$ \\
\hline $\begin{array}{l}\text { Latency of } \\
\text { First Response }\end{array}$ & 4.40 & 4.01 & 5.43 \\
\hline $\begin{array}{l}\text { Time per } \\
\text { Shuttle }\end{array}$ & .654 & .616 & .609 \\
\hline
\end{tabular}

Fixed Ratio 
the second study, CS termination and withholding of the US was contingent upon the occurrence of a fixed number of responses. This procedure is referred to here as fixed ratio avoidance responding.

\section{Procedure}

The same basic procedure was used in Study 2 as in Study 1 for avoidance learning. Following successful acquisition of the avoidance responses, $(85 \%$ for four experimental sessions) fixed ratio reinforcement of $2: 1$ was introduced, i. e., the Ss had to hurdle the barrier twice before the CS terminated and shock prevented. Fixed ratios were gradually increased with the restriction that the animal be capable of maintaining $85 \%$ successful avoidance after the third experimental session. If criterion was not satisfied the ratio was reduced until the criterion was attained. The $\mathrm{S}$ was discontinued if it failed to reach criterion after three attempts to increase the ratio size.

\section{Hesults and Discussion}

Eight Ss acquired the continuously reinforced avoidance response; two Ss reached criterion for a 2:1 ratio but failed to attain a higher ratio; four Ss acquired a 4:1 ratio before failing to meet criterion, and two Ss went through a 7:1 and 10:1 fixed ratio. One of the two latter Ss attained a fixed ratio of $21: 1$.

The latency of the first response in the ratio run and the average shuttles per second are found in Table I for the two Ss that reached a 10:1 ratio. Before computing shuttles per second it was first necessary to correct for the apparent differences in latency for the various ratios. This was done by subtracting the latency (time interval from the onset of the CS to the first ratio response) of the first response from the total time, i. e., from onset of CS to the last response in the ratio run, and dividing by the size of the ratio minus one. It can be seen from Table I that average rate of responding tends to increase with increases in the size of the ratio. Although not systematically related to ratio size, the latency of the first response is greater for the 10:1 ratio than for either the $4: 1$ or $7: 1$ ratio. Both of the latter findings suggests that behavior maintained under fixed ratio avoidance has properties similar to behavior maintained under fixed ratio reinforcement in the appetitive situation. Failure to find greater similarity may be due to the gross shuttle response required in this experiment as contrasted with the more refined bar pressing response usually used in the appetitive situation. In any event this study has shown that stable avoidance responding can be maintained over long periods and that intermittent reinforcement of the avoidance response is feasible.

\section{References}

COONS, E. E., ANDERSON, N. H., \& MYERS, A. K. Disappearance of avoidance responding during continued training. J.comp. physiol. Psychol., 1960, 53, 290-292.

MEYER, D. R., CHO, C., \& WESEMANN, ANN S. On problems of conditioning lever-press avoidance responses. Psychol. Rev., 1960, 67, 224-228. 